\title{
Churermodell - Möglichkeit, um Hoffnung zu schaffen
}

\author{
Eine Unterrichtsanlage, die hilft, den Ansprüchen an \\ Ausbildung und Bildung gerecht zu werden
}

DOI: https://doi.org/10.53349/sv.2021.i3.a131

\begin{abstract}
Was in der Stadt Chur als Schulentwicklung begann, hat unterdessen in vielen anderen Schulen Einzug gehalten: Das Churermodell. Was das Churermodell ist und warum dies als Modell für eine Hoffnungsschule dienen kann, erklärt die Autorin in diesem Beitrag.
\end{abstract}

Churermodell, Unterrichtsentwicklung, personalisiertes Lernen, Schulentwicklung

Würde ich ein Schulsystem für eine Hoffnungsschule kreieren, wäre dies eine Schule, die Kinder und Jugendliche im Grundsatz darin unterstützt, zu werden, wer sie im Wesen schon sind. Sie würde den Kindern und Jugendlichen Raum bieten, ihre Talente zu entdecken, ihre Fähigkeiten zu bilden, ihre Fertigkeiten zu erproben und so als gereifte und mutige Persönlichkeiten ihr Leben und die Welt (mit) zu gestalten. Ein Schwerpunkt der Ausbildung liege in der Persönlichkeitsbildung und der Förderung des persönlichen Ausdrucks in den vier Dimensionen, in denen ein Mensch sich zum Ausdruck bringen kann: In der Wort-Sprache (darunter auch die Mathematik), der Ton-Sprache, der Körper-Sprache, der Produkt-Sprache. Die Schule würde es den Kindern also ermöglichen, „,sich all das anzueignen, was sie brauchen, damit sie ihr Leben so gestalten können, dass es gelingt. [...] Und das, was sie dazu benötigen, und was wir ihnen dafür mit auf den Weg geben können, ist Bildung. Bildung für ein gelingendes Leben. Alles andere ist Ausbildung." (Hüther, G. et al., 2020, S. 18). Die Hoffnungsschule, die zukunftsfähig sein soll, muss sich also sowohl nach dem Erwerb von Wissen, Können und Kompetenzen richten, als auch der Bildung dieser jungen Menschen an sich verschreiben. Doch wie kann dieser Spagat gelingen? 


\section{Öffnung des Unterrichts}

Unabdingbar ist aus meiner Sicht die grundsätzliche Öffnung des Unterrichts, weit mehr als dies bereits heute oft praktiziert wird. Peschel (2005) definiert offenen Unterricht in allen grundlegenden Dimensionen: organisatorisch, methodisch, inhaltlich, sozial. Im Unterschied zum traditionellen Unterricht werden dementsprechend folgende Punkte von den Schüler*innen selbst geregelt:

- Die individuellen fachlichen und überfachlichen Lerninteressen der Kinder bestimmen das Lerngeschehen und darüber hinaus auch

- das soziale Geschehen und

- die über die Lerngruppe/Klasse hinausgehenden Interaktionen - auch die ausserschulischen.

Peschel $(2005,5.78)$ nennt in seiner Definition von offenem Unterricht folgende Merkmale und Qualitätskriterien:

Offener Unterricht gestattet es dem Schüler, sich unter Freigabe von Raum, Zeit und Sozialform Wissen und Können innerhalb eines „offenen Lehrplans“ an selbst gewählten Inhalten auf methodisch individuellem Weg anzueignen. Offener Unterricht zielt im sozialen Bereich auf eine möglichst hohe Mitbestimmung, bzw. Mitverantwortung des Schülers bezüglich der Infrastruktur der Klasse, der Regelfindung innerhalb der Klassengemeinschaft sowie der gemeinsamen Gestaltung der Schulzeit ab.

Auch der bekannte Entwicklungsforscher Remo H. Largo fordert in seinem Buch Das passende Leben (2017, S.133):

Ein Bildungswesen, das der Chancengerechtigkeit verpflichtet ist, bietet nicht nur einer kleinen Elite ein hohes Ausbildungsniveau, sondern wird möglichst allen Menschen gerecht, indem es die Vielfalt aller Begabungen berücksichtigt. In einem derartigen Bildungssystem wird nicht mehr erwartet, dass alle Schüler gleich gute Leistungen erbringen und langfristig gleich hohe Kompetenzen erwerben. Es verhilft vielmehr allen Kindern gemäss ihren individuellen Begabungen zum grösstmöglichen schulischen Erfolg und stellt so Bildungsgerechtigkeit dar. [...] Genauso wichtig wie die Übereinstimmung zwischen Herausforderung und Entwicklungsstand ist, dass das Kind selbstbestimmt lernen darf. Dafür gibt es zwei gewichtige Gründe. Zum einen entwickelt jedes Kind seine eigenen Lernstrategien, wie es Gelerntes mit seinen bestehenden Fähigkeiten und dem vorhandenen Wissen am besten verknüpfen kann. Zum anderen werden Lernerfahrungen nur dann nachhaltig verinnerlicht, wenn sie mit den bereits erworbenen Fähigkeiten, Fertigkeiten und dem vorhandenen Wissen verwoben werden können. « (Largo, 2017, S. 161)

Auch der Lehrplan 21 des Kantons Graubünden formuliert in seinen Grundlagen die Bildungsziele folgendermassen:

Bildung ist ein offener, lebenslanger und aktiv gestalteter Entwicklungsprozess des Menschen. Bildung ermöglicht dem Einzelnen, seine Potenziale in geistiger, kultureller und lebenspraktischer Hinsicht zu erkunden, sie zu entfalten und über die Auseinandersetzung mit sich und der Umwelt eine eigene Identität zu entwickeln. Bildung befähigt zu einer eigenständigen und selbstverantwortlichen Lebensführung, die zu verantwortungsbewusster und selbstständiger Teilhabe und 


\section{\# schuleverantworten}

führungskultur_innovation_autonomie

Mitwirkung im gesellschaftlichen Leben in sozialer, kultureller, beruflicher und politischer Hinsicht führt. (Lehrplan $21 \mathrm{GR}, 2016)$.

Dementsprechend muss sich die öffentliche Volksschule wandeln und sich weiter öffnen, sonst ist das vorgegebene Ziel nicht zu erreichen.

Eine mögliche Unterrichtsanlage, um dem Auftrag der Vermittlung von Ausbildungsinhalten sowie der Bildung an sich gerecht zu werden, bietet das Churermodell (Thöny, 2019). Dieses Modell ist darauf ausgerichtet, kindgerecht einen individualisierenden und differenzierenden Unterricht umzusetzen. Peter Lienhard $(2012$, S.14) von der Hochschule für Heilpädagogik $(\mathrm{HfH})$ in Zürich schreibt:

Das Bestechende am „Churermodell“ ist, dass es die Basis für viele wesentliche Entwicklungen der heutigen Schule legt - sei es individualisierte Lernförderung, inklusive Schulungsform und integrative Begabungs- und Begabtenförderung.

\section{Das Churermodell als mögliche Grundlage für offenen Unter- richt}

Das Churermodell entstand 2010 aus Bemühungen der Stadtschule Chur (Graubünden, Schweiz), den Kindern einen besseren Übergang vom Kindergarten in die Schule zu ermöglichen. Dazu wurde die Unterrichtsanlage des Kindergartens auf die Schule übertragen. Es sind vor allem folgende vier Elemente, die allen Beteiligten Orientierung geben: Die Gestaltung des Schulzimmers, Inputs im Kreis, Lernen mit Lernaufgaben, freie Wahl des Arbeitsplatzes und des*der Lernpartner*in.

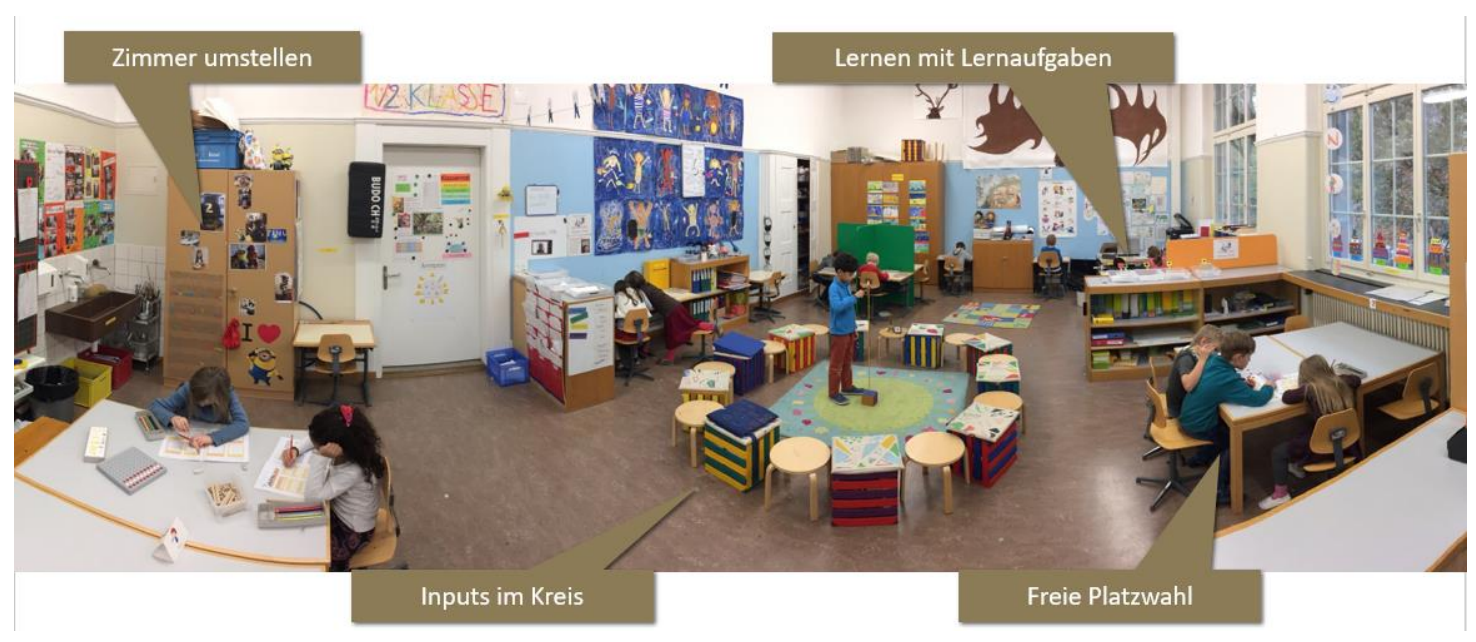

Abbildung 1: Elemente des Churermodells (Thöny 2019) 


\section{Grundsatz: Der Raum arbeitet ,pädagogisch‘ mit}

Wer im Churermodell unterrichten will, muss bereit sein, das Schulzimmer umzustellen. Die Schulbänke sind nicht mehr zur Wandtafel oder Leinwand hin ausgerichtet. Den persönlichen Arbeitsplatz der Schüler*innen gibt es nicht mehr. Das Schulmaterial wird von allen in Gestellen aufbewahrt. Es gibt Einzelarbeitsplätze, solche, die sich für Partnerarbeiten eignen, und Gruppenarbeitsplätze. Einige Arbeitsplätze sind nach aussen gerichtet und sorgen für weniger Ablenkung. Andere Arbeitsplätze sind frei im Raum angelegt und eignen sich für das Zusammenarbeiten. Wichtig ist, genügend Verkehrsfläche zu erhalten, damit sich die Schüler*innen im Schulzimmer ohne zusätzliches Konfliktpotenzial bewegen können.

\section{So viel Instruktion wie nötig, so viel Konstruktion wie möglich}

Der Sitzkreis ist im Churermodell von zentraler Bedeutung. Es ist der Ort der Gemeinschaft, an dem man sich zu Beginn und am Ende des Unterrichts trifft. Jede Lektionseinheit wird zudem mit einem Input im Kreis eröffnet. Dieser soll nicht länger als 15 Minuten dauern. In dieser Zeit werden die Lernaufgaben vorgestellt und Voraussetzungen geschaffen, damit die Schüler*innen über längere Zeit an den Inhalten arbeiten können. Hier soll der Grundsatz lauten, so viel Instruktion wie nötig und so viel Konstruktion wie möglich. Die Phase der Instruktion wird zugunsten der Phase der Konstruktion kurzgehalten. Jede*r Schüler*in hat im Sitzkreis einen fest zugewiesenen Platz.

Instruktion

Konstruktion

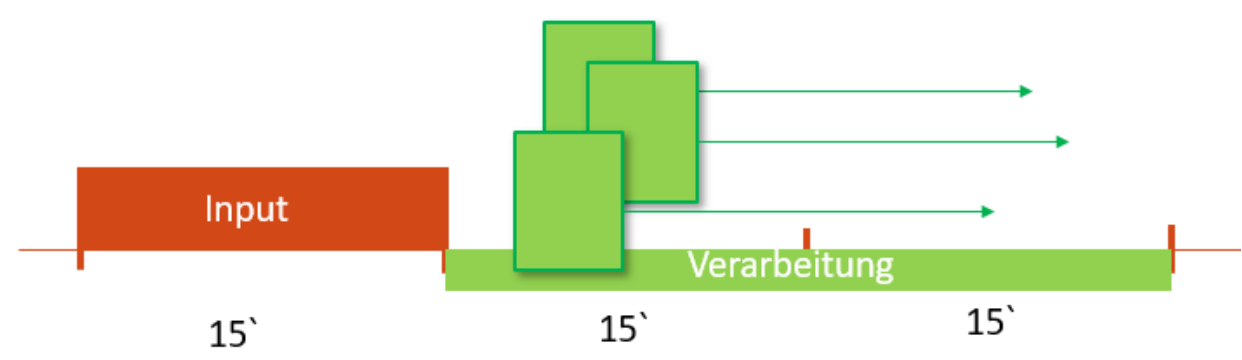

Abbildung 2: Zeitliche Gestaltung der Lektion (Thöny 2019)

\section{Selbsteinschätzung des eigenen Leistungsniveaus}

Die Rolle der Lehrkraft besteht darin, Lernerwartungen zu formulieren, Grundanforderungen transparent zu machen und die Schüler*innen im Lernen zu unterstützen. Die Lehrpersonen 


\section{\# schuleverantworten}

konzipieren in Zusammenarbeit mit involvierten Schulischen Heilpädagog*innen und/oder Fachlehrpersonen den Unterricht in Lernaufgaben. Sie lösen sich damit vom traditionellen Lehrverständnis und schaffen so Möglichkeiten zur Differenzierung. Lernen geschieht somit durch Auseinandersetzung mit bedeutsamen Lernaufgaben auf unterschiedlichen Anspruchsniveaus und mit adäquater Lernbegleitung. Schüler*innen sind aufgefordert, jene Lernaufgaben zu wählen, die ihrem aktuellen Lernstand entsprechen. Dazu müssen sie ihr Leistungsniveau mit Blick auf die Aufgabenschwierigkeit einschätzen. Hattie bezeichnet dies in seinen Forschungen zu „Lernen sichtbar machen“ als „,wirkungsvollste aller untersuchten 150 unterrichtlichen Massnahmen" (Hattie, 2015, S.49ff).

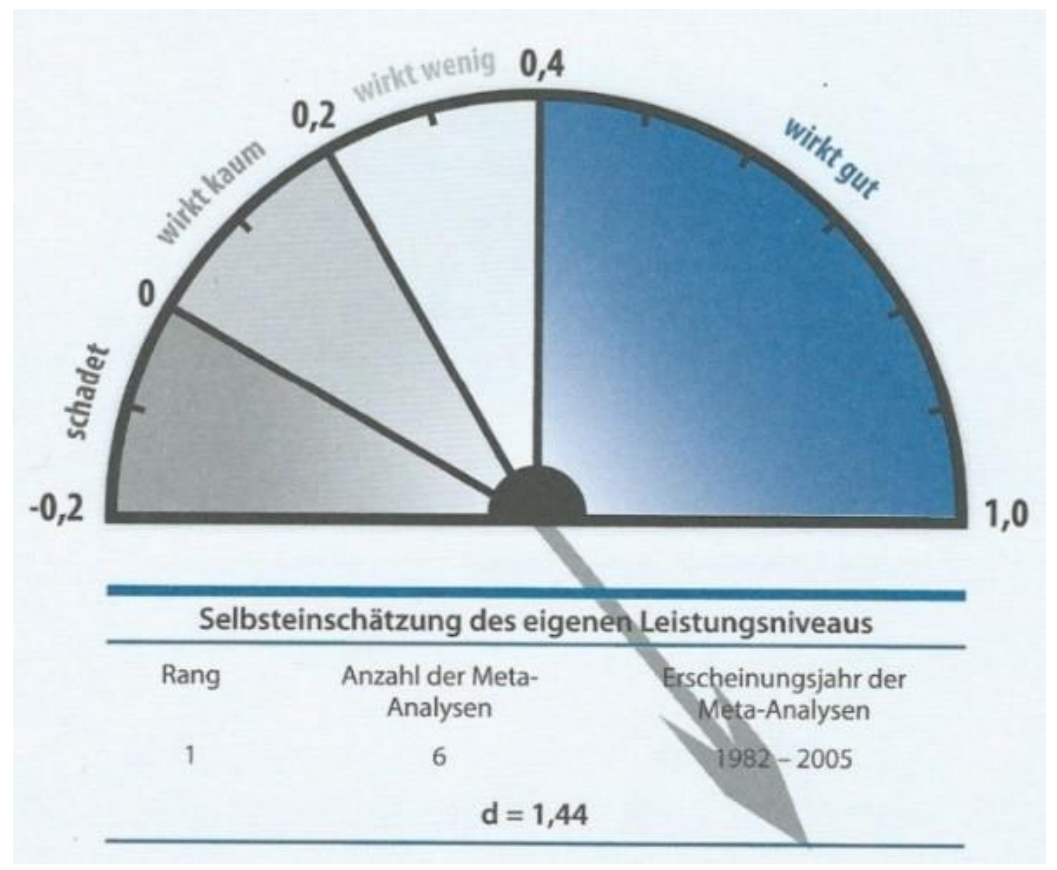

Abbildung 3: Selbsteinschätzung des eigenen Leistungsniveaus (Zierer 2014)

Damit wird das Lernen personalisiert: Die Lernenden stellen einen Bezug von sich zur Sache her. Das didaktische Dreieck kann für das Churermodell wie folgt adaptiert werden: 


\section{\# schuleverantworten}

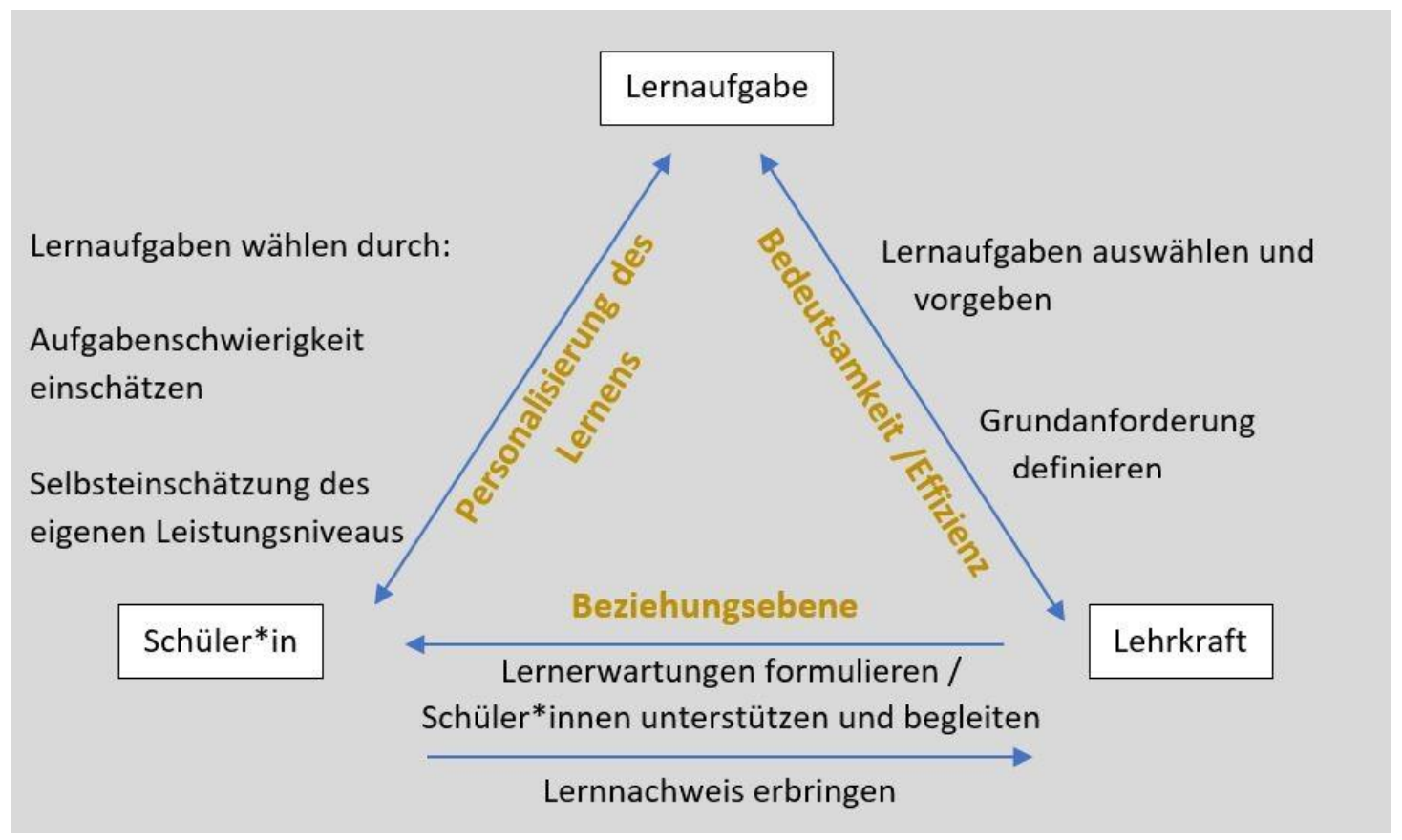

Abbildung 4: Didaktisches Dreieck im Churermodell (Thöny 2020)

\section{Wahl des Lernortes und des Lernpartners}

Schüler*innen sind im Churermodell aufgefordert, ihre Lernsituation mitzubestimmen. Damit werden die Kinder zunehmend "Subjekt des schulischen Lernens" (Hüther, 2016, S. 70), was sich einerseits positiv auf die Motivation auswirkt, andererseits Anforderungen an die Selbststeuerung stellt. Freie Platzwahl bedeutet jedoch nicht, sich einfach hinzusetzen, wo man will: Es geht darum, einen Lernort zu finden, wo man gut arbeiten kann. Damit ist klar, dass sich für jede neue Arbeit die Frage nach dem geeigneten Arbeitsplatz oder dem*der Lernpartner*in stellt. Erstaunt stellen viele Lehrpersonen fest, dass sich die freie Platzwahl beruhigend auf den Unterricht auswirkt (Thöny, 2019). Dies könnte daran liegen, dass Schüler*innen mit gemeinschaftsbelastendem Verhalten im Churermodell die „Bühne“ entzogen wird. Zudem schliessen die unterschiedlichen Ausrichtungen der Arbeitsplätze viele Ablenkungen aus. Die Partizipation der Schüler*innen am Unterricht fördert überfachliche Kompetenzen. Das Mass an Fremdsteuerung durch die Lehrperson nimmt ab, je mehr die Schüler*innen Selbststeuerung und damit Verantwortung übernehmen können.

\section{Öffnung des Unterrichts im Churermodell}

Unser jeweiliges Handeln ist stark von normativen Überzeugungen geleitet, die auf gemachten Erfahrungen beruhen. Mit dem Wechsel zum Churermodell ermöglichen sich Lehrperso- 
nen neue Erfahrungen, da sich das Handlungsspektrum der Schüler*innen durch die Wahlmöglichkeiten vom „Müssen und Sollen“ auf das „Dürfen und Wollen“ erweitert. Das Churermodell erfordert zudem, dass die Lehrpersonen ihr Vertrauen in die Lernfähigkeit der Kinder stärken (Pool Maag, 2017). Dieses Vertrauen wächst mit jeder positiven Erfahrung. Aus neurobiologischer Sicht ist es unmöglich, „zu leben ohne zu lernen“ (Hüther, 2016. S.121). Lehrpersonen müssen sich zurücknehmen und trotzdem die Leitung behalten. Dabei spielt das Beobachten, das weit mehr als „Sehen“ ist, eine wichtige Rolle. Der Lernerfolg hängt nämlich stark von einer adäquaten Lernbegleitung ab, die individuell, zum richtigen Zeitpunkt und in der richtigen Dosierung erfolgen muss. Lehrpersonen erhalten so die Möglichkeit, stets ihre Kompetenzen selbstwirksam im Führen von Lerngesprächen auszubauen.

Die Verbindung von Binnendifferenzierung und Öffnung wirkt sich positiv auf das Lern- und Sozialverhalten aus. Lehrpersonen schaffen im Churermodell Zeit und Raum für die individuelle Lernbegleitung und erhalten so jederzeit einen guten Überblick über Lernstand und Förderbedarf der Schüler*innen. Die Partizipation wirkt sich positiv auf die Motivation der Lernenden aus. Das Churermodell ist ein Modell, das die Steuerung schulischen Arbeitens sowohl durch Lehrer*innen als auch durch Schüler*innen ermöglicht und zudem die Verbindung von instruktionalem und explorativem Lernen zulässt. Es erfordert jedoch, dass Lehrpersonen ihr Vertrauen in die Lernfähigkeit der Schüler*innen stärken (Pool Maag, 2020).

\section{Fazit}

Was die heranwachsenden Kinder und Jugendlichen vor allem brauchen, ist eine gelungene Bildung, denn das Wissen als Produkt der Ausbildung ist heutzutage fast überall verfügbar. Es braucht meiner Meinung nach Akteure und Fakten, die zu einer gelingenden Bildung beitragen können. Es braucht Schulleitungspersonen, die auf strategischer und operativer Ebene gute Bildung ermöglichen und unterstützen, es braucht Lehrpersonen, die den Mut zur Öffnung des Unterrichts haben, die diese Bildung mit Inhalt füllen und die vor einer engeren Beziehung zu Schüler*innen nicht zurückschrecken, und es braucht Schüler*innen, die sich mit ebendieser Bildung Wissen und Kompetenzen aneignen wollen. Ferner sind geklärte Strukturen notwendig, damit die Bildung nicht mit Verwaltungshindernissen zu kämpfen hat. Im Kerngeschäft der Bildung, nämlich im Unterricht, braucht es klar definierte Prozesse, um Entwicklungen in Gang zu bringen oder in Schwung zu halten. Weiter braucht es Unterstützung aller Beteiligten durch Behörden und Institutionen, um eine vielfältige Bildung zu ermöglichen. Und es braucht letztendlich den Mut und den Willen, genau hinzuschauen, zu evaluieren und zu reflektieren, um Bestehendes weiter zu optimieren. All diese Faktoren tragen dazu bei, dass die Schule sich wandeln und Nährboden genug bieten kann, damit die „ausgeschulten" Kinder und Jugendlichen sich in einer Welt zurechtfinden können, die sich seit einigen Jahrzehnten in dramatischem Wandel befindet. Denn in einer zeitgmässen Schule wurden sie ausreichend gebildet und damit befähigt, dieses Leben gelingend zu leben. Ich habe Hoffnung, dass dies möglich sein wird und dass die Hoffnungsschule bald schon die Norm sein wird. 


\section{Literaturverzeichnis}

Hattie, J.A.C. (2015): Lernen sichtbar machen. Überarbeitete deutschsprachige Ausgabe von „Visible learning“, besorgt von Beywl, W. \& Zierer, K. Baltmannsweiler: Schneider Verlag Hohengehren (3., erweiterte Auflage).

Hüther, G. (2016). Mit Freude lernen ein Leben lang. Göttingen: Vandenhoeck \& Ruprecht.

Hüther, G., Heinrich, M., \& Senf, M. (2020). \#Education für future. Bildung für ein gelingendes Leben. München: Goldmann.

Largo, Remo H. (2017). Das passende Leben. Was unsere Individualität ausmacht und wie wir sie leben können. Frankfurt/M.: S. Fischer.

Lehrplan 21 GR (2016). Verfügbar unter https://gr-d.lehrplan.ch/index.php?code=e|200|1

Lienhard, P. (2012). Geht die Begabtenförderung in der integrativen Schule auf oder unter? In Begabungsförderung integriert. Band II. Zürich: Stiftung für hochbegabte Kinder.

Peschel, F. (2005). Offener Unterricht. Idee, Realität, Perspektive und ein praxiserprobtes Konzept in der Evaluation. Teil I. Baltmannsweiler: Schneider Verlag Hohengehren.

Pool Maag, S. (2017). Das Churermodell. Einblicke in eine Didaktik für inklusive Lerngruppen. Schweizerische Zeitschrift für Heilpädagogik 23 (5-6), S. 32-39. Verfügbar unter http://www.churermodell.ch/images/sampledata/downloads/Pool_Maag_1705-0632.pdf

Pool Maag, S. (2020). Ergebnisfeedback zu Projekt «Link -Lernen in inklusiven Lerngruppen». Das Churermodell der Binnendifferenzierung -eine Basis für das Lehren und Lernen in inklusiven Lerngruppen? Zürich: Pädagogische Hochschule.

Thöny, R. (2019). Churer Modell - eine Möglichkeit der Binnendifferenzierung im Unterricht. Verfügbar unter www.churermodell.ch/attachments/article/1/Broschüre\%20Churermodell.pdf

\section{Autorin}

Karin Lutz-Bommer, M.A.

Schulentwicklung, ausgebildete Primarlehrerin und Schulleiterin, seit 2021 Leiterin von Kursen und Lehrgängen im Prorektorat Weiterbildung und Dienstleistung der PH Graubünden, Chur, Schweiz. Davor 13 Jahre tätig als Primarlehrerin 4.-6. Klasse in St. Gallen und 1.-6. Klasse in Tenna und 6 Jahre als Schulleiterin auf der Stufe Sek1 in Chur.

Kontakt: karin.lutz@phgr.ch 